\title{
Die Global Education Week - Ein handlungsstarkes europaweites Netzwerk
}

Die jährlich im November stattfindende Global Education Week verbindet Akteurinnen und Akteure aus dem Bildungsbereich zu einem vielgestaltigen Netzwerk. Die europaweite Aktionswoche widmet sich Themen des Globalen Lernens und fand zuletzt vom 18. bis 24. November 2019 unter dem Motto „Wake Up! Letzter Aufruf für den Klimaschutz“ statt. Die GlobalEducation Week ist eineInitiative des North-South-Center des Europarates in Lissabon und wird in Deutschland von der Informationsstelle Bildungsauftrag Nord-Süd beim World University Service (WUS) koordiniert.

2019 nahmen deutschlandweit 40 Organisationen mit vielfältigen Angeboten teil, die ebenso großes Engagement wie Kreativität verrieten: So organisierten die Fachabiturientinnen und -abiturienten des Hermann-Gmeiner-Berufskollegs in Moers ein veganes-vegetarisches Buffet für die ganze Schule; die Industrie- und Handelskammer Südthüringen veranstaltete eine regionale Energiekonferenz; die Volkshochschule Bremen bot eine Bildungswoche zu den 17 Nachhaltigkeitszielen der Agenda 2030 an; der Allgemeine Studierendenausschuss (AStA) der Hochschule für Wirtschaft und Recht in Berlin rief eine Klimaschutzwoche aus.

Das Ziel der Aktionswoche ist es, die Themen des Globalen Lernens, insbesondere die Nachhaltigkeitsziele der Agenda 2030, einer breiten Öffentlichkeit zu vermitteln. Schulen, Universitäten und Bildungsinitiativen aller Art sind dazu eingeladen, sich mit Beiträgen zu beteiligen. Das offene Format sieht Informationsveranstaltungen ebenso wie Videoclips, Ausstellungen oder Unterrichtseinheiten vor, die von den teilnehmenden Initiativen eigenverantwortlich durchgeführt werden. Das Herzstück der Kampagne ist die vom WUS betreute Website www.globaleducation.de, die die vielfältigen Beiträge vorstellt, Interessierte informiert und zu eigenen Aktivitäten inspiriert. Auf diese Weise entsteht ein höchst aktives Netzwerk, das Initiativen aus der Landespolitik, dem Bildungsbereich und der Zivilgesellschaft verbindet. Wie gut dieser Austausch zwischen nicht-staatlichen und staatlichen Akteurinnen und Akteuren funktioniert, zeigt der Hinweis auf die Aktionswoche auf der Website des Bundesministeriums für Umwelt, Naturschutz und nukleare Sicherheit in der Rubrik „Umwelt im Unterricht“. Die europaweiten Aktivitäten präsentiert das North-South-Center auf seiner Website, wodurch die Netzwerkmitglieder ihre Strategien und Ressourcen teilen können. Im „Global Education Network“ sind zivilgesellschaftliche Organisationen aus Ländern wie Estland, Dänemark, Frankreich, Marokko, Serbien und der Türkei vertreten. Auch die
Entscheidung für das Motto „Wake Up! Letzter Aufruf für den Klimaschutz" wurde gemeinsam beschlossen. Das Thema traf nicht zuletzt deshalb auf große Zustimmung, weil ökologische Themen auch in Ländern mit rechtspopulistischen Regierungen die Öffentlichkeit erreichen.

Die deutsche Kampagne konnte zugleich an eine Initiative von 118 zivilgesellschaftlichen Organisationen anschließen, die sich im Vorfeld des UN-Gipfels zur Umsetzung der Agenda 2030 formiert hatte. In einem offenen Brief riefen die Unterzeichnenden im Mai 2019 die Bundesregierung dazu auf, entschiedener für die Erreichung der Ziele der Agenda 2030 einzutreten. Sie betonten die Dringlichkeit einer umwelt- und klimaschonenden Wende in der Energie-, Verkehrs-und Agrarpolitik und forderten Maßnahmen, um die weltweite Ungleichheit innerhalb und zwischen Staaten zu bekämpfen.

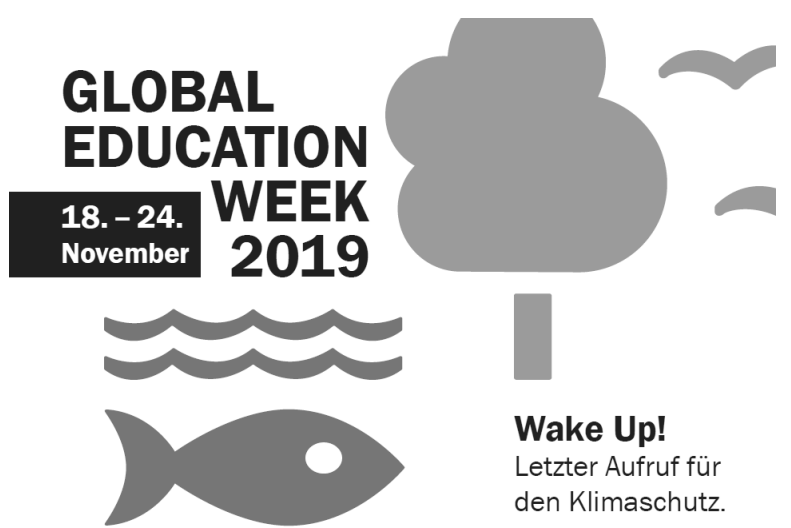

Abb. 1: Flyer der Global Education Week 2019, Quelle: WUS Germany

Im November 2020 wird die Global Education Week erneut stattfinden. Sie wird sich dem Thema „Youth Engagement“ widmen und damit wichtige Impulse aus dem Vorjahr aufnehmen. Im Zuge der weltweiten Proteste für den Klimaschutz gingen auch in Europa zehntausende Jugendliche auf die Straße und traten damit als wichtige politische Akteurinnen und Akteure der Gegenwart und der Zukunft in Erscheinung.

Dr. Ursula Grünenwald

Informationsstelle Bildungsauftrag Nord-Süd beim World University Service (WUS) - Deutsches Komitee e. V., infostelle@wusgermany.de, www.wusgermany.de \& www.globaleducationweek.de doi.org/10.31244/zep.2020.01.09 